\title{
Absorption and emission properties of photonic crystals and metamaterials
}

by

\author{
Lili Peng \\ A thesis submitted to the graduate faculty \\ in partial fulfillment of the requirements for the degree of \\ MASTER OF SCIENCE \\ Major: Condensed Matter Physics \\ Program of Study Committee: \\ Costas M. Soukoulis, Major Professor \\ Rana Biswas \\ Jiming Song \\ Edward $\mathrm{Yu}$
}

Iowa State University

Ames, Iowa

2007

Copyright (C Lili Peng, 2007. All rights reserved 
UMI Number: 1446140

\section{UMI}

UMI Microform 1446140

Copyright 2008 by ProQuest Information and Learning Company.

All rights reserved. This microform edition is protected against unauthorized copying under Title 17, United States Code.

ProQuest Information and Learning Company 300 North Zeeb Road

P.O. Box 1346

Ann Arbor, MI 48106-1346 


\section{TABLE OF CONTENTS}

LIST OF FIGURES $\quad$ iii

ABSTRACT $\quad$ iv

CHAPTER 1. INTRODUCTION 1

CHAPTER 2. ABSORPTION AND TRANSMISSION PROPERTIES OF PC 6

2.1 Introduction of Absorption and Transmission Calculations 6

2.2 Simulation Results 8

CHAPTER 3. THERMAL EMISSION

3.1 Introduction of Thermal Emission 15

3.2 Thermal Emission Properties for Two Structures 15

CHAPTER 4. SPONTANEOUS EMISSION IN PHOTONIC CRYSTALS 17

4.1 Introduction of Spontaneous Emission in PC 17

4.2 Simulation Results 17

CHAPTER 5. SUMMARY AND DISCUSSION 22

$\begin{array}{ll}\text { BIBLIOGRAPHY } & 23\end{array}$

ACKNOWLEDGEMENTS 25 


\section{LIST OF FIGURES}

Figrue 1.1 Illustration of one-dimensional (1D), two-dimensional (2D) and threedimensional (3D) Photonic Crystals 3

Figure 2.1 Absorption spectra for woodpile PC of TE mode, with $a=4.2 \mu \mathrm{m}, w=1.2 \mu \mathrm{m} \quad 7$

Figure 2.2 Absorption spectra for woodpile PC of TM mode, with $a=4.2 \mu \mathrm{m}, w=1.2 \mu \mathrm{m} \quad 8$

Figure 2.3 Structure of a photonic crystal with 8 layers of metal rods in a square lattice with the top layer of rods are cutted into half, 3/4 and 5/6 10

Figure 2.4 Absorption and absorption ratio spectra 11

Figure 2.5 Structure of further modified PC 12

Figure 2.6 Absorption spectra for full and cutted structrues 12

Figure 2.7 Electric field at $\omega a / 2 \pi c=0.52$

Figure 2.8 Current distribution $J=(1-1 / \varepsilon) \partial E / \partial t \quad 13$

Figure 2.9 Joule heating $\quad 13$

Figure 2.10 Periodic structure with the same parameters as the cutted-surface structure 14

Figure 2.11 Absorption spectra of the periodic structure using Comsol Multiphysics and Ansoft HFSS

Figure 3.1 Emission Intensity for Woodpile Structure at $T=625 \mathrm{~K} \quad 16$

Figure 3.2 Emission Intensity for Cutted Structure at $T=625 K \quad 16$

Figure 4.1 Structure of a 2D photonic crystal 18

Figure 4.2 Absorption, Reflection and Transmission Spectra for $\Gamma X$ direction 19

Figure 4.3 Absorption, Reflection and Transmission Spectra for $\Gamma M$ direction 19

Figure 4.4 Emission for normalized frequency $0.5 \quad 20$

Figure 4.5 Emission for normalized frequency 0.57 20

Figure 4.6 Emission of line current source from 2D PC 21

Figure 4.7 Normalized (to that of the vacuum) emission of line current source from 2D PC 21 


\section{ABSTRACT}

We study the emission and absorption properties of photonic crystals and metamaterials using Comsol Multiphysics and Ansoft HFSS as simulation tools.

We calculate the emission properties of metallic designs using drude model and the results illustrate that an appropriate termination of the surface of the metallic structure can significantly increase the absorption and therefore the thermal emissivity.

We investigate the spontaneous emission rate modifications that occur for emitters inside two-dimensional photonic crystals and find the isotropic and directional emissions with respect to different frequencies as we have expected. 


\section{CHAPTER 1. INTRODUCTION}

For centuries, people are looking for ways to control the properties of materials. Advances in metallurgy, ceramics and plastics have enablesd us to control the mechanical properties of materials; advances in semiconductor physics have enabled us to control the conducting properties. In the last decades, advances in photonic crystals (PCs) have enabled us to control the optical properties of materials.

A crystal with a periodic arrangement of atoms or molecules presents a periodic potential to an electron propagating through it. The periodic potential in a semiconductor crystal affects the electron motion by defining allowed and forbidden electronic energy bands due to Bragg-like diffranction from the atoms, so that electrons are forbidden to propagate with certain energies in certain directions. If the potential is strong enough, the gap might extend to all possible directions, resulting in a complete band gap.

Photonic crystals are composed of periodic dielectric or metallic-dielectric structures that are designed to affect the propagation of electromagnetic waves in the same way as the semiconductor affects the propagation of electrons. Consequently, photons in PC can have band structures, localized defect modes, surface modes, etc. This new ability to mold and guide light leads naturally to many novel phenomena associated with light. The absence of allowed propagating EM modes inside the structures, in a range of wavelengths called a photonic band gap (PBG), gives rise to distinct optical phenomena such as inhibition of spontaneous emission and low-loss waveguides. Of particular interest is a PC whose bandstructure possesses a complete photonic band gap. A complete photonic band gap defines a range of frequencies for which light is forbidden to propagate in all directions.

It was Lord Rayleigh [1] who first studied the electromagnetic wave propagation in a one-dimensional periodic structure, such as a multilayer film, and showed that any such one- 
dimensional system has a band gap. In the case where the dielectric constant periodically varies spatially in one direction, we call it one-dimensional (1D) photonic crystal. Such onedimensional periodic structure is the simplest form of photonic crystals. One dimensional periodic systems continued to be studied extensively and many applications appeared, such as reflective coatings where the reflection band corresponds to the photonic band gap and distributed feedback (DFB) diode lasers where a crystallographic defect is inserted in the photonic band gap to define the laser wavelength. We define 2D and 3D PCs in a similar way, corresponding to the respective cases where dielectric constant varies along two and three independent directions. 100 years after Rayleigh, in 1987, Eli Yablonovitch [2] and Sajeev John [3] for the first time pointed out and demonstrated independently that one can manipulate light propagation more freely by using two- and three-dimensional PCs. Eli Yablonovitch showed by simulation that in photonic band gap, all modes are suppressed in all directions, hence spontaneous emission corresponding to the gap energy is inhibited inside a 3D PC. Meanwhile, Sajeev John claimed that new phenomena can be observed by using a PC, which includes localization of light. These papers have evoked great interest of many research groups around the world.

In the first four or five years after 1987, most theoretical studies were focused on finding out the most suited structures and the refractive indices (n) needed for obtaining a full PBG in 2D or 3D PC. In the latter half of the 1990s, people gradually became aware that many interesting phenomena don't need to have 2D or 3D full PBG to be observed. Experimentally, a variety of photonic band structures were examined by using samples with lattice constant a in the millimeter and submillimeter region. Eli Yablonovitch and his coworkers verified in 1991 that a 3D sample with lattice constant a in micrometer region also has a full PBG [4]. In the near-infrared or optical region, a 3D structure with a full PBG, was still technically difficult to fabricate until around 1999, when Noda and his coworkers for the first time succeeded in fabricating such a sample [5]. 

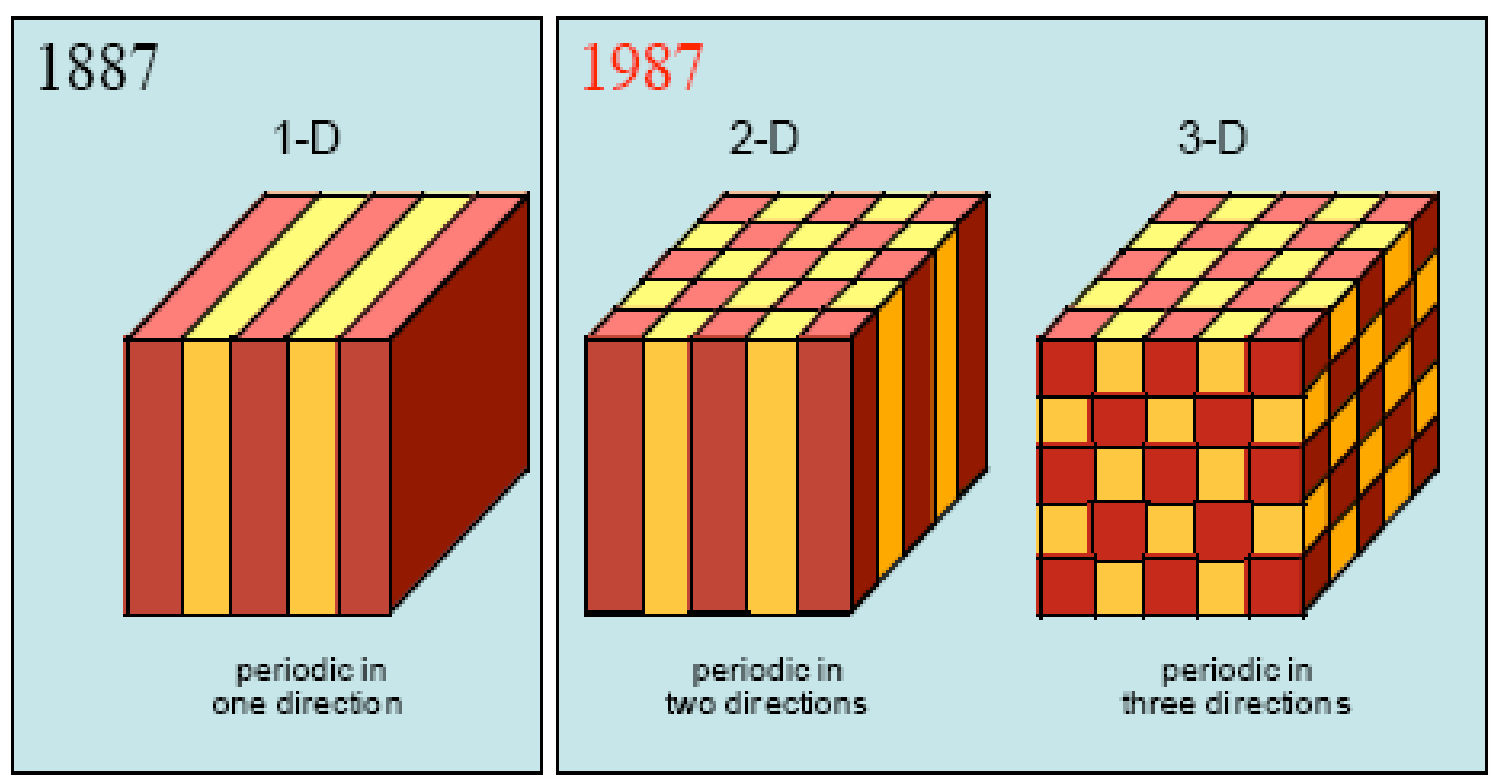

Figrue 1.1 Illustration of one-dimensional (1D), two-dimensional (2D) and threedimensional (3D) Photonic Crystals

Natural photonic crystals exist. A prominent example of a photonic crystal is the gemstone opal. Its play of variable colours is essentially a photonic crystal phenomenon based on Bragg diffraction of light on the crystal's lattice planes. Another well-known photonic crystal is found on the wings of some butterflies such as those of genus Morpho [6]. The tails of peacock also have PC structures which makes them shining and colorful [7].

In terms of fabrication, the major challenge for higher dimensional photonic crystals is to have sufficient precision to prevent scattering losses blurring the crystal properties and have robustly mass-production processes. One promising method for fabrication of twodimensionally periodic photonic crystals is a photonic-crystal fiber. Using fiber draw techniques developed for communications fiber it meets these two requirements. For three dimensional photonic crystals various techniques have been used. Layer-by-Layer Lithography can be used to make rods \& holes simultaneously. Wafer Fusion technique is used to fabricate woodpile structures [7]. Micromanipulation can be used in stacking process [8]. To circumvent nanotechnological methods with their complex machinery, alternate 
approaches have been followed to grow photonic crystals as self-assembled structures from colloidal crystals.

Our projects focus on the emission properties of photonic crystals and metamaterials. The environment of an excited atom is known to influence its rate of spontaneous emission. Spontaneous emission of an atom, dipole or current source, suppresssed for photons in the photonic band gap and enhanced in specific frequency regions, demonstrate that the interaction between the radiation field and matter can be designed and modified using photonic crystals. For the spontaneous emission of an emitter embedded in a photonic crystal, the rate of emission is a function of the frequency of the emitter, the emitter's position and orientation as well as the geometric and material parameters of the photonic crystal.

Photonic crystals consisting of metallic materials exhibit a number of interesting properties if properly designed. One-dimensional photonic crystals composed of a stack of alternationg layers of metallic and dielectric materials can be designed to be transparent over a range of optical frequencies [9] [10]. These structures can also be designed to enhance reflection [11] or absorption [12]. In addition, three-dimensional metallic photonic crystals can exhibit absorption suppression in the photonic band gap and strong absorption enhancement at the photonic band edge [13] [14]. A finite two-dimensional square lattice of metallic cylinders in air can be designed to have great absorptance over a wide optical wavelength range and for a wide range of incidence angles [15]. If a system is designed such that the coupling of the resonances of spherical particle sembedded near the surface of a metal and the surface plasmon is very strong, the coupling of the resonances will lead to enhanced absorption at selected frequencies. Such systems can be employed to modify or control thermal emission [16]. Thermal radiation is just spontaneous emission that is thermally driven and is in thermal equilibrium with its material surroundings. By optimizing the coupling of the radiation field of a PBG material and a spatially extended collection of atomic or electronic emitters, it is possible to achieve dramatic modifications of Planck's 
blackbody radiation spectrum [17] [18]. M. Florescu and J. P. Dowling prove that the central quantity that determines the thermal radiation characteristics is the area of the iso-frequency surface and not the photonic density of states as it is generally assumed.

This thesis is organized as follows. In Chapter 2, we analyse the absorption, transmission and reflection properties for structures with metallic and dielectric materials. By modifying the surface of a photonic crystal structure, we're able to achieve great enhancement in absorption. In Chapter 3, we use the structures specified in Chapter 2 to calculate thermal emissions. Our results prove that the blackbody radiation can be greatly modified. In Chapter 4, spontaneous emission in two-dimensional photonic crystal is discussed. Simulation results are given and discussed. The summary and conclusions of this thesis are presented in Chapter 5. 


\section{CHAPTER 2. ABSORPTION AND TRANSMISSION PROPERTIES OF PC}

\subsection{Introduction of Absorption and Transmission Calculations}

We use Comsol Multiphysics and Ansoft HFSS as simulation tools, solving for stationary solutions in frequency domain.

For a 2D photonic crystal with metallic rods standing in air, we use the Drude model in our simulation. The dielectric function of the metallic rods is given by:

$$
\varepsilon(\omega)=1-\omega_{p}^{2} /\left(\omega^{2}+i \gamma \omega\right) \text {, with } \omega_{p}=1419 T H z, \quad \gamma=134.9 T H z .
$$

For siplicity, let's consider 1D photonic crystal with plane wave propagation on-axis, normal to the surfaces. Let $T$ and $R$ be the ratios of transmitted and reflected optical power with respect to an incident flux normalized to unity. If the material is lossless, the conservation of energy requires that $T+R=1$. If the material is lossy, we define absorption as $A=1-T-R$. From Kirchoff's second law, we know that absorptance equals to emittance if the source is a perfect blockbody, independent of the nature of the material [20, 21, 22]. We can as well extend the theory to $2 \mathrm{D}$ and $3 \mathrm{D}$ photonic crystals.

For example, the absorption spectra for the woodpile structure PC are shown in Figure 2.1 (for TE mode) and Figure 2.2 (for TM mode) respectively. The structure is arranged so that the rods are orthogonal to each other in adjacent layers. These layers follow an $A B C D$ pattern, such that $C$ is the same as A shifted by half a lattice constant, and the same is true for $\mathrm{D}$ and $\mathrm{B}$. B is the same as A rotated by $90^{\circ}$. Absorption spectra with different thickness of the layers are compared. The structure with layers of thickness $6 \mu m$ has the biggest absorption. 


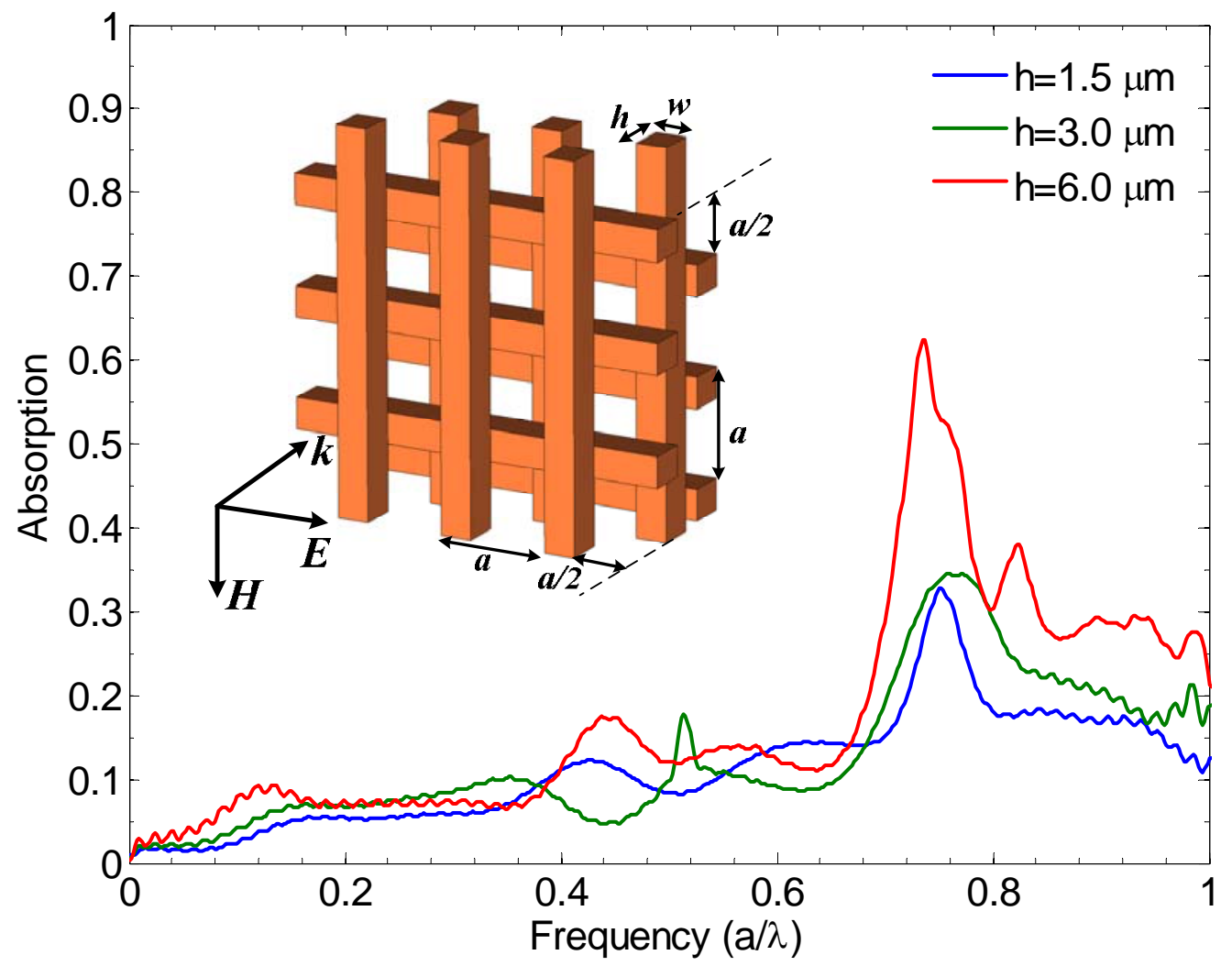

Figure 2.1 Absorption spectra for woodpile PC of TE mode, with $a=4.2 \mu \mathrm{m}, w=1.2 \mu \mathrm{m}$ 


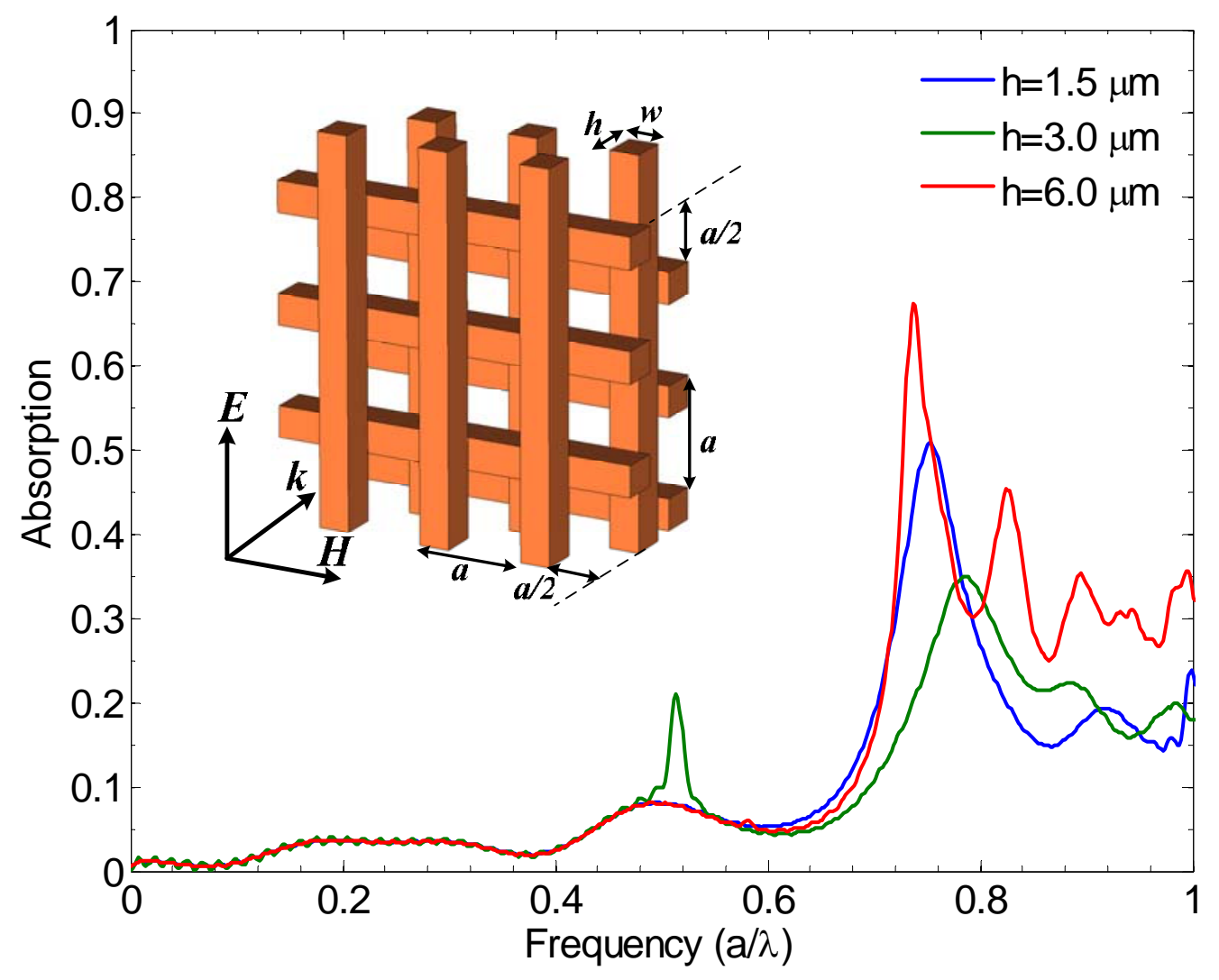

Figure 2.2 Absorption spectra for woodpile PC of TM mode, with $a=4.2 \mu \mathrm{m}, w=1.2 \mu \mathrm{m}$ 


\subsection{Simulation Results}

For a one-dimensional PC with 8 layers of metal rods in a square lattice (lattice constant a, discretized by 10 grid points) with radius $r=0.2 a$. The light propagation direction is normal to the interfaces and the light is polarized along the rods. In order to obtain maximum absorption, the top layer of rods are cutted into half, 3/4 and 5/6. See Figure 2.3 as the structure.

The absorption spectrum (Figure 2.4 top) shows that when the top and bottom layers of rods are cutted into half, we get the largest absorption. In the other hand, the absorption ratio spectrum (Figure 2.4 bottom) shows the absorption ratio $A /(1-R)$, namely the absorption capability of the photonic crystal, remains almost unchanged. We can conclude that by modifying the surface, the bulk absorption of the PC doesn’t change. The reflection is reduced, hence the total absorption increases as more wave energies enters the PC. 

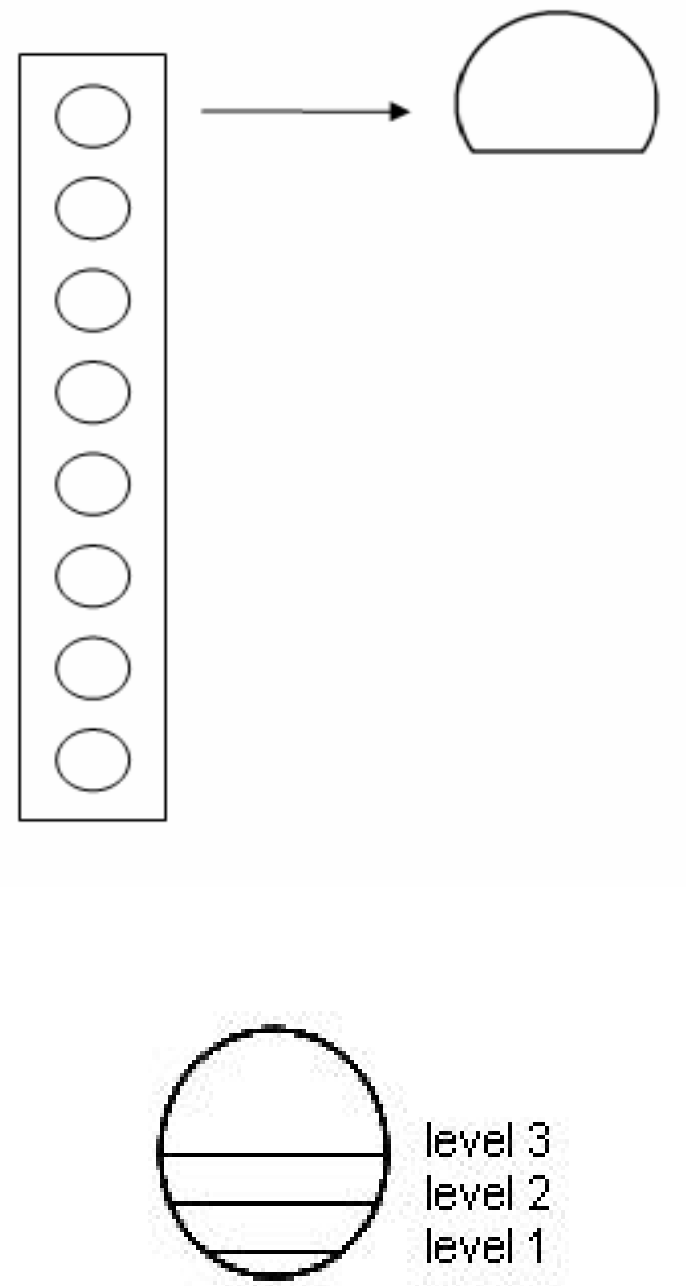

Figure 2.3 Structure of a photonic crystal with 8 layers of metal rods in a square lattice with the top layer of rods are cutted into half, $3 / 4$ and 5/6 


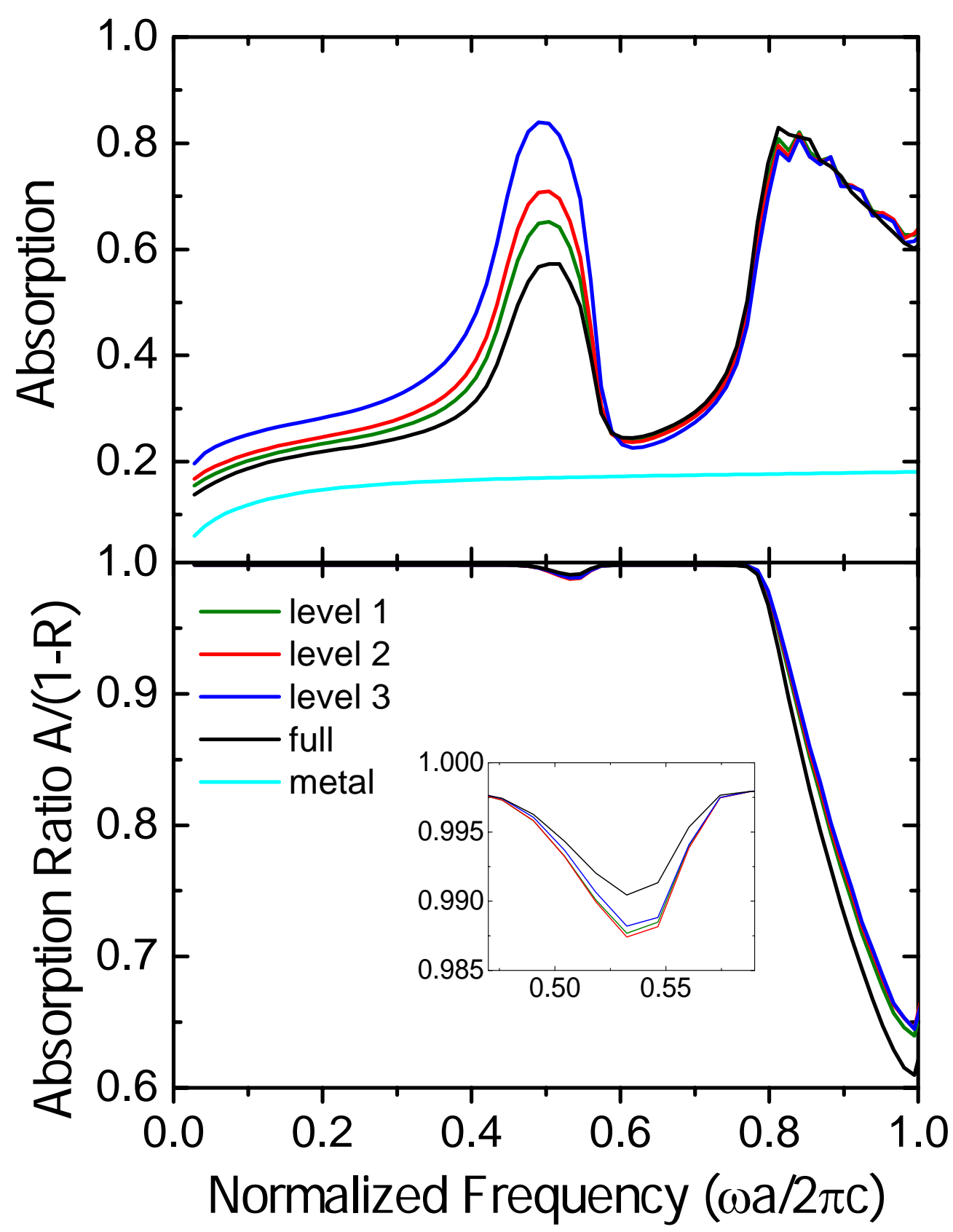

Figure 2.4 Absorption and absorption ratio spectra 
Now we consider the same structure except that the first two layers of metallic rods are both cutted into half (Figure 2.5), the absorption at a specific frequency can be increased to 0.98 , compared to the absorption of the non-cutted structure which is only about 0.5 , shown in Figure 2.6. The electric field, current distribution and joule heating at normalized frequency 0.52 are shown in Figure 2.7, 2.8 and 2.9 respectively.

By modifying PC surfaces, we're able to make great improvements in impedance matching, thus dramatically increases the absorption.

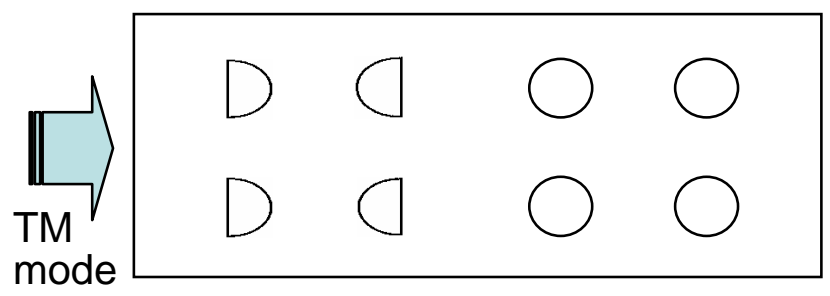

Figure 2.5 Structure of further modified PC

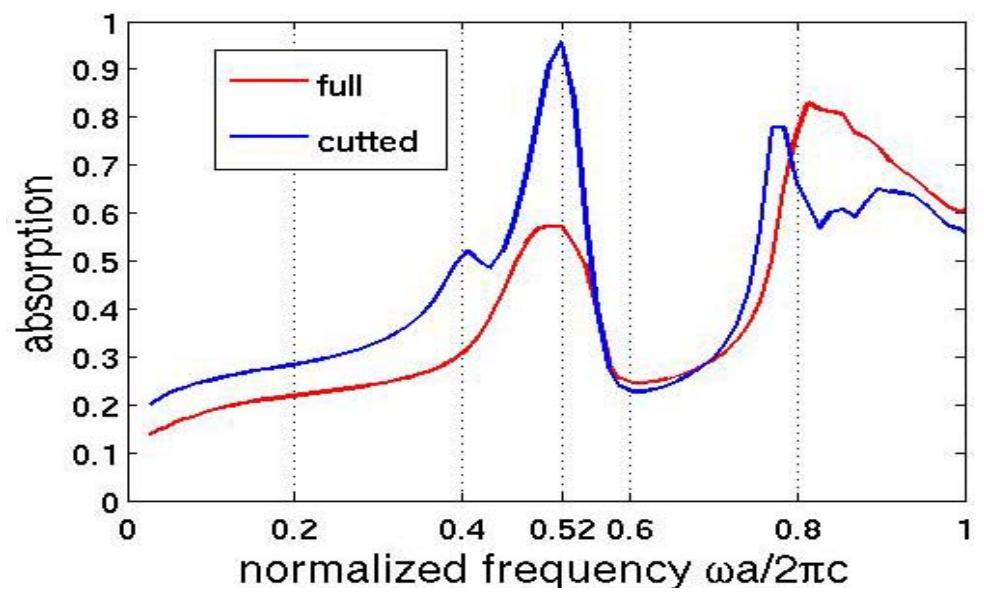

Figure 2.6 Absorption spectra for full and cutted structrues 


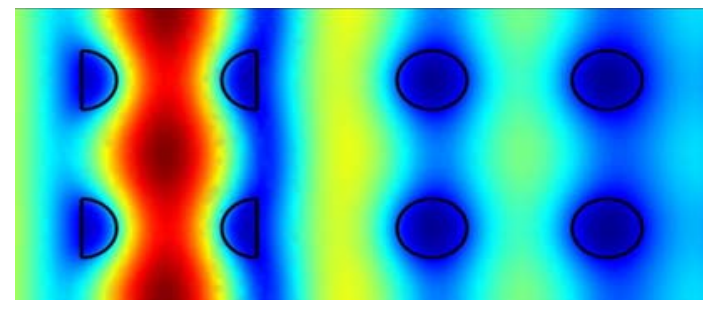

Figure 2.7 Electric field at $\omega a / 2 \pi c=0.52$

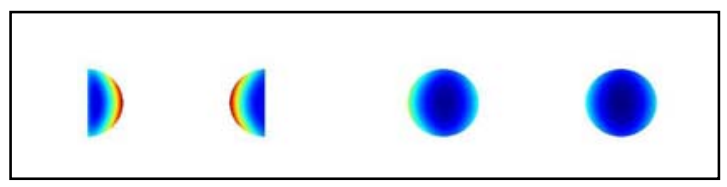

Figure 2.8 Current distribution $J=(1-1 / \varepsilon) \partial E / \partial t$

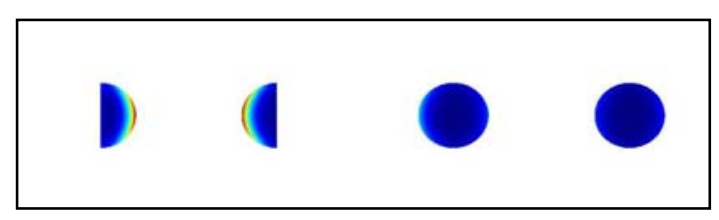

Figure 2.9 Joule heating 
A periodic structure (Figure 2.10) is also examined. Comsol Multiphysics and Ansoft HFSS simulation results are almost the same except a small frequency shift (Figure 2.11). The impedence of the this structure is not as small as the surface-cutting structure.

$\begin{array}{lllllllllll}0 & 0 & 0 & 0 & 0 & 0 & 0 & 0 & 0 & 0 \\ 0 & 0 & 0 & 0 & 0 & 0 & 0 & 0 & 0 & 0 \\ 0 & 0 & 0 & 0 & 0 & 0 & 0 & 0 & 0 & 0 \\ 0 & 0 & 0 & 0 & 0 & 0 & 0 & 0 & 0 & 0 \\ 0 & 0 & 0 & 0 & 0 & 0 & 0 & 0 & 0 & 0\end{array}$

Figure 2.10 Periodic structure with the same parameters as the cutted-surface structure

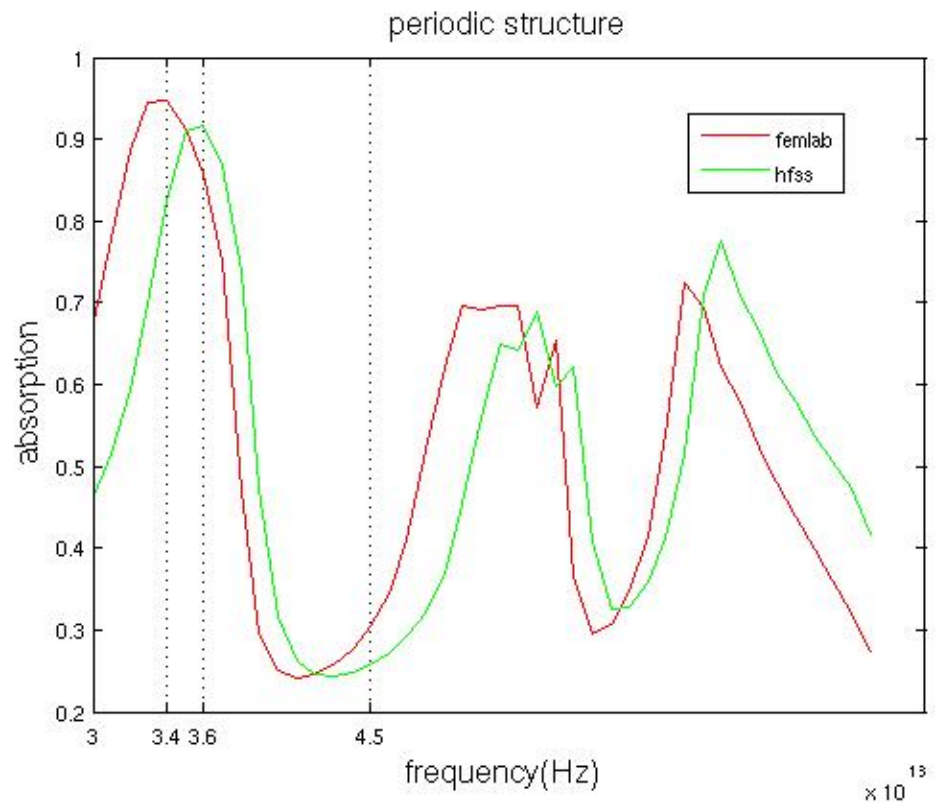

Figure 2.11 Absorption spectra of the periodic structure using Comsol Multiphysics and Ansoft HFSS 


\section{CHAPTER 3. THERMAL EMISSION}

\subsection{Introduction of Thermal Emission}

A blackbody is defined as an object of perfect absorption. In practice, most objects have only finite absorption which are called graybodies. Kirchhoff' s law states that for an object in thermal equilibrium with the surrounding radiation field, its Absorptivity equals to Emissivity. Joannopoulos’ group [19] have proven that $I(\omega, T)=I_{0}(\omega, T) * A(\omega)$, where $I(\omega, T)$ is radiation intensity, $I_{0}(\omega, T)$ is free-space blackbody radiation intensity and $A(\omega)$ is

absorbance. Thus, by manipulate the absorbance, the thermal emission profile can be modified or tailored by means of altering the geometry or the materials used for the system. Thermal radiation from 2D photonic crystals has been studied within the contexts of spectral and directional control [23,24,25,26,27].

\subsection{Thermal Emission Properties for Two Structures}

We can obtain the radiation power of a structure by taking the absorbance spectra that we have calculated previously and multiplying them by the blackbody radiation spectrum.

Let's consider the woodpile structure whose absorption is less than 0.5 and the firsttwo-layers-cutted structure whose absorption can be as big as 0.98 at a specific frequency. For the woodpile structure, at temperate $625 \mathrm{~K}$, we can see an emission pick shown in Figure 3.1. For the surface-cutted structure, the emission intensity curve almost touches the ceiling of the blackbody radiation intensity profile at a specific frequency (Figure 3.2). We show the emission spectrum of a blackbody for comparison. 
This surface-cutted structure enables us to achieve $98 \%$ of the emission of a blackbody. We can shift the peak by changing the lattice constant of the structure. Thus it's possible to place strong emission peaks at multiple frequencies.

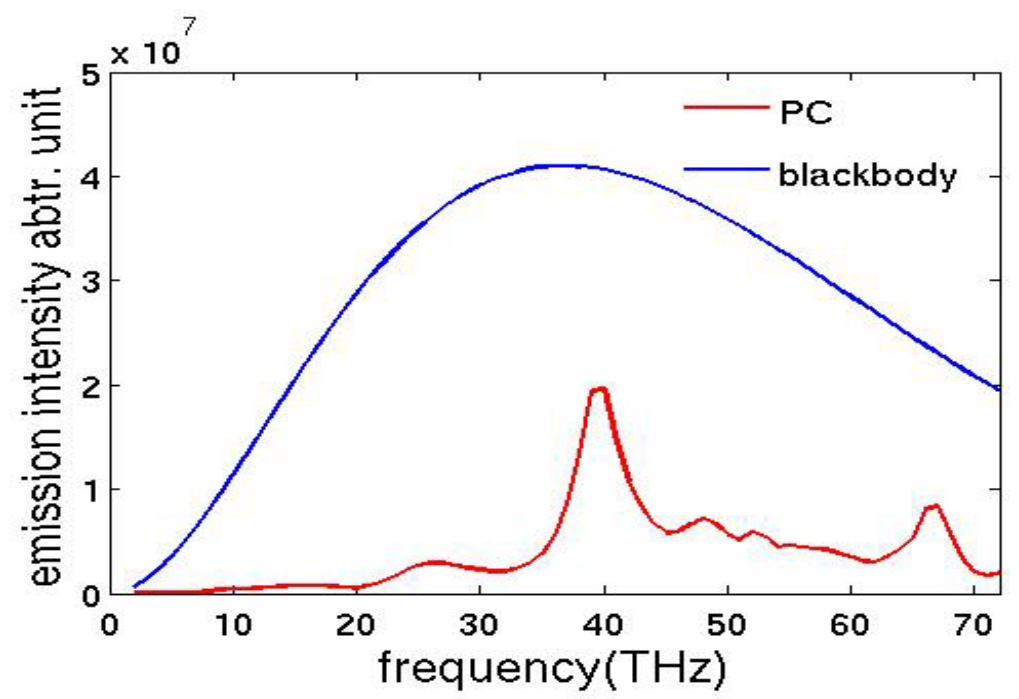

Figure 3.1 Emission Intensity for Woodpile Structure at $T=625 \mathrm{~K}$

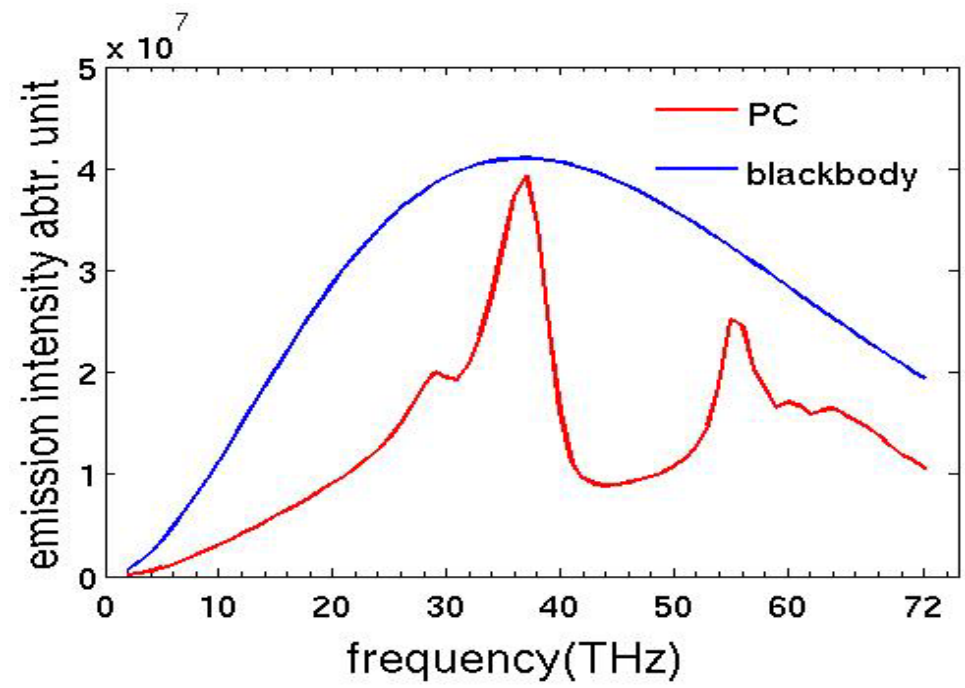

Figure 3.2 Emission Intensity for Cutted Structure at $T=625 \mathrm{~K}$ 


\section{CHAPTER 4. SPONTANEOUS EMISSION IN PHOTONIC CRYSTALS}

\subsection{Introduction of Spontaneous Emission in PC}

The environment of an emitter has influence on its rate of spontaneous emission. A photonic crystal can alter the density of states surrouding the emitter. If the density of states is zero, then the spontaneous emission is prohibited. If the density of states is increased respective to that of free space, the spontaneous emission is enhanced. By controlling the interaction between the emitter and the field, many interesting phenomena can be observed.

\subsection{Simulation Results}

We use Comsol Multiphysics and Ansoft HFSS as simulation tools. They solve for stationary solutions in frequency domain.

For a 2D photonic crystal with metallic rods standing in air, we use the Drude model in our simulation. The dielectric function of the metallic rods is given by:

$$
\varepsilon(\omega)=1-\omega_{p}^{2} /\left(\omega^{2}+i \gamma \omega\right) \text {, with } \omega_{p}=1419 T H z, \quad \gamma=134.9 T H z .
$$

The 2D PC structure is shown in Figure 4.1. The absorption, transmission and reflection spectra with respect to two different directions are shown in Figure 4.2 and 4.3. The light propagation direction is normal to the interfaces and the light is polarized along the rods. The shaded areas are band gaps. We can see that in $\Gamma X$ direction, the frequency range is from 0.4 to 0.57 for band, and from 0 to 0.4 and from 0.57 to 0.75 for gaps. In $Г M$ direction, the frequency range is from 0.4 to 0.72 for band, and from 0 to 0.4 and from 0.72 to 0.78 for gaps. The normalized frequency 0.5 is in the band for both $\Gamma X$ and $\Gamma M$ 
directions of the crystal; whereas the normalized frequency 0.57 is in the gap for $\Gamma X$ direction and in the band for the $\Gamma M$ direction.

An infinite long line current source radiates in the middle of this 2D PC, and the spontaneous emission of this emitter is modified by this 2D PC. Radiation boundary condition is used for the boundaries of the 2D photonic crystal.

We can see the emission pattern with respect to different frequencies in Figure 4.4 and 4.5. When the normalized frequency $\omega a / 2 \pi c$ equals to 0.5 , the emission pattern is approximately isotropic; when the normalized frequency $\omega a / 2 \pi c$ equals to 0.57 , the emission is directional.

The total power output is calculated and shown in Figure 4.6 with the normalized emission ( to that of the vacuum) shown in Figure 4.7.

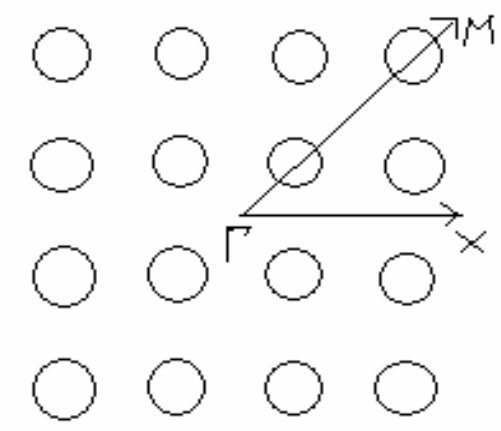

Figure 4.1 Structure of a 2D photonic crystal 


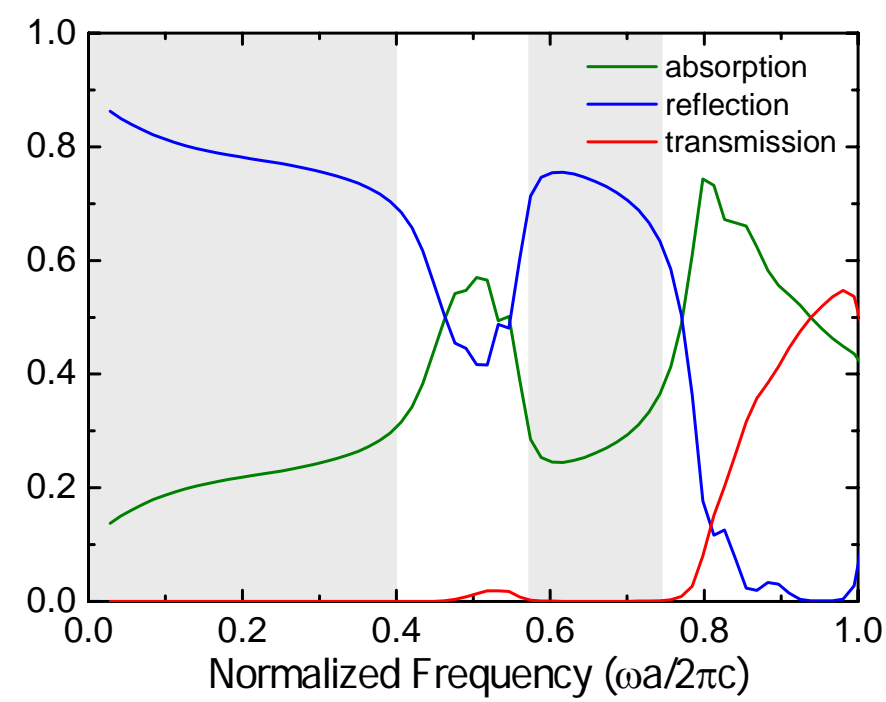

Figure 4.2 Absorption, Reflection and Transmission Spectra for $\Gamma X$ direction

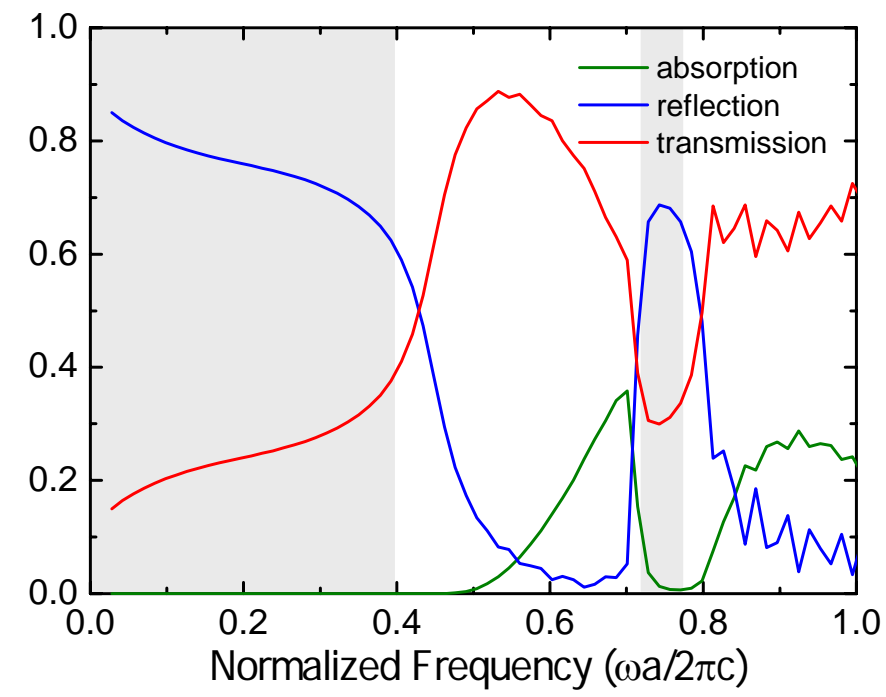

Figure 4.3 Absorption, Reflection and Transmission Spectra for $\Gamma M$ direction 


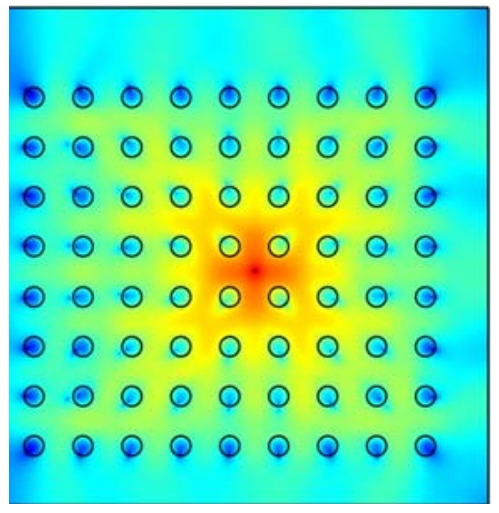

Figure 4.4 Emission for normalized frequency 0.5

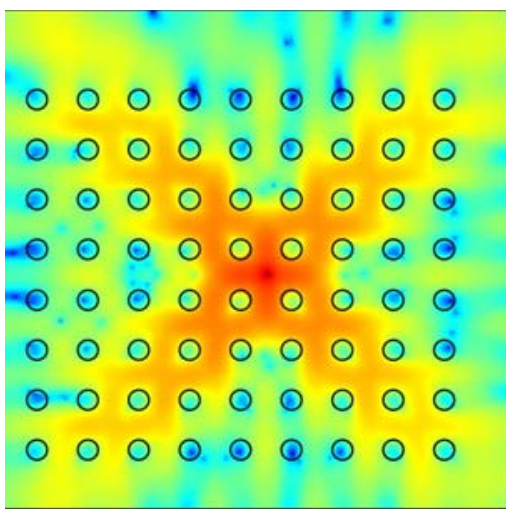

Figure 4.5 Emission for normalized frequency 0.57 


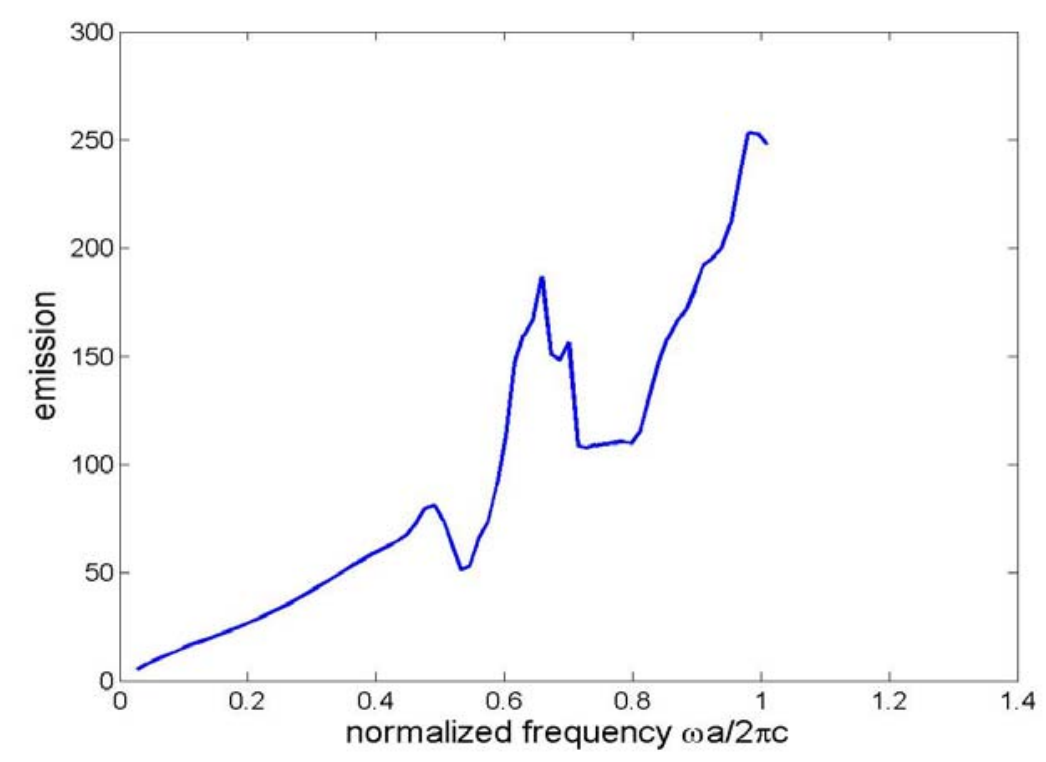

Figure 4.6 Emission of line current source from 2D PC

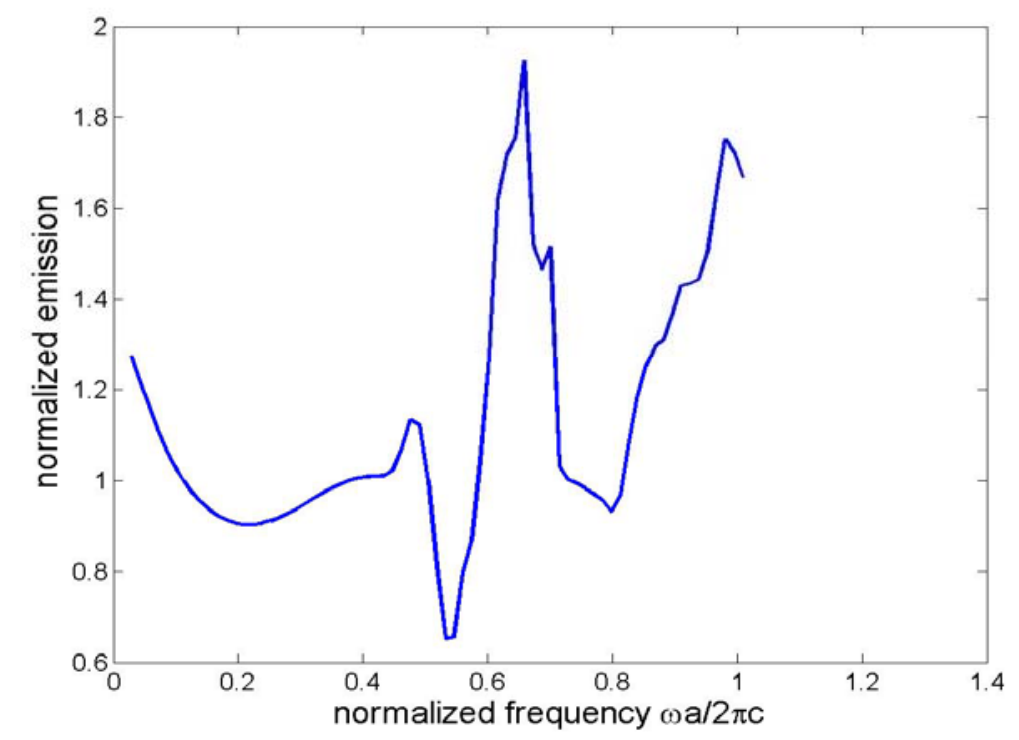

Figure 4.7 Normalized (to that of the vacuum) emission of line current source from 2D PC 


\section{CHAPTER 5. SUMMARY AND DISCUSSION}

Using commertial simulation tools of Comsol Multiphysics and Ansoft HFSS, we investigate the transmission, refletion and absorption properties of different structures. The transmission and reflection are obtained directly from the energy flow into and out of the PC bulk, while the absorption is obtained by using the rules of energy conservation.

We are aiming at the structure which can give the maximum thermal emission. From Kirchoff's second law, we know that the ratio of the thermal emittance to the absorptance is a constant, independent of the nature of the material. Now our goal turns to searching for the structure with big absorption. After modifying the surface of a 2D metallo-dielectric photonic crystal, we find that the absorption of the structure is greatly enhanced due to the improvement in impedence matching. More energies enter into the system, hence more are absorbed. If the above system is heated to $625 K$, the emission intensity will be $98 \%$ of the emission of a blackbody when normalized frequeny equals to 0.52 .

We are also interested in how photonic crystals manipulate the spontaneous emission of an emitter inside the PC. We know that in 1D photonic crystal, the spontaneous emission ratio depends on the position and oriention of the emitter, as well as the geometry and materials used for the structure [28]. The emission will be suppressed in gaps and enhanced at band edges. For 2D photonic crystal, we've seen isotropic and directional emissions in different region of frequencies. We also calculate the total power output and the normalized power output of this system with respect to different frequencies. 


\section{BIBLIOGRAPHY}

[1] J. W. S. Rayleigh, Phil. Mag. 26, 256-265 (1888).

[2] E. Yablonovitch, Phys. Rev. Lett., Vol. 58, 2059 (1987).

[3] S. John, Phys. Rev. Lett. 58, 2486 (1987).

[4] E. Yablonovitch, T. J. Gmitter and K. J. Leung, Phys. Rev. Lett. 67, 2295 (1991).

[5] S. Noda, K. Tomoda, N. Yamamoto, and A. Chutinan, Science, 289, 604 (2000).

[6] S. Kinoshita, S. Yoshioka and K. Kawagoe, Proc. R. Soc. Lond. B 269, 1417-1421 (2002).

[7] S. Noda et al., Science 289, 604 (2000).

[8] K. Aoki et al., Appl. Phys. Lett. 81 (17), 3122 (2002).

[9] M. Scalora, M. J. Bloemer, A. S. Pethel, J. P. Dowling, C. M. Bowden, and A. S. Manka, J. Appl. Phys. 83, 2377 (1998).

[10] M. J. Bloemer and M. Scalora, Appl. Phys. Lett. 72, 1676 (1998).

[11] A. J. Ward, J. B. Pendry, and W. J. Stewart, J. Phys.: Condens. Matter 7, 2217 (1995).

[12] J. Yu, Y. Shen, X. Liu, R. Fu, J. Zi, and Z. Zhu, J. Phys.: Condens. Matter 16, L51 (2004).

[13] S. Y. Lin, J. Moreno, and J. G. Fleming, Appl. Phys. Lett. 83, 380 (2003).

[14] S. Y. Lin, J. G. Fleming, Z. Y. Li, I. EI-Kady, R. Biswas, and K. M. Ho, J. Opt. Soc. Am. B 20, 1538 (2003).

[15] G. Veronis, R. W. Dutton and S. Fan, J. of Appl. Phys. 97, 093104 (2005).

[16] G. Sun and C. T. Chen, Phys. Rev. E 73, 1 (2006).

[17] C. M. Cornelius and J. P. Dowling, Phys. Rev. A 59, 4736 (1999).

[18] S. Y. Lin, J. Moreno, and J. G. Fleming, Appl. Phys. Lett. 83, 380 (2003).

[19] C. Luo, A. Narayanaswamy, G. Chen, and J. D. Joannopoulos, Phys. Rev. Lett. 93, 213905 (2004). 
[20] M. Planck, The Theory of Heat Radiation (Dover, New York, 1959); T. S. Kuhn, Black-Body Theory and the Quantum Discontinuity, 1894-1912 (University of Chicago Press, Chicago, 1987)

[21] P. Pigeat, D. Rouzel, and B. Weber, Phys. Rev. B 57, 9293 (1998).

[22] J. B. Marion, Classical Electromagnetic Radiation (Academic Press, New York, 1965), Sec. 6.5.

[23] A. Mekis, A. Dodabalapur, R. E. Slusher, and J. D. Joannopoulos, Opt. Lett. 25, 942 (2000).

[24] H. Sai, H. Yugami, Y. Akiyama, Y. Kanamori, and K. Hane, J. Opt. Soc. Am A 18, 1471 (2001).

[25] M. U. Pralle, N. Moelders, M. P. McNeal, I. Puscasu, A. C. Greenwald, J. T. Daly, E. A. Johnson, I. EI-Kady, and R. Biswas, Appl. Phys. Lett. 81, 4685 (2002).

[26] A. Enoch, J.-J. Simon, L. Escoubas, P. Torchio, and G. Albrand, Appl. Phys. Lett. 86, 261101 (2005).

[27] M. Laroche, R. Carminati, and J.-J. Greffet, Phys. Rev. Lett. 96, 123903 (2006).

[28] Adan S. Sanchez and P. Halevi, Phys. Rev. E 72, 056609 (2005). 


\section{ACKNOWLEDGEMENTS}

I would like to give my sincerest thanks to my advisor Prof. Costas M. Soukoulis for his guidence, patience and support throughout this research. I'm grateful to him for giving me this exciting and challenging project, helping and guiding me through the most difficult times of this work. My gratitude also goes to Dr. Thomas Koschny for his kind help and guidence throughout the project.

I would also like to express my gratitude to my committee members Prof. Rana Biswas, Jiming Song and Edward Yu for helping me with various aspects of research and studies. I learned a great lot from Prof. Jiming Song and Prof. Edward Yu’s lectures. The discussion with Prof. Rana gave me inspirations in my work. In addition, I thank Jiangfeng Zhou for his valuable help and discussions, especially in the initial stage of the project. 